\title{
Análise de prescrições destinadas ao emagrecimento em farmácia magistral antes e após a vigência da RDC No 52/2011
}

\author{
Evaluation of prescriptions of pharmaceuticals used \\ to weight reduction prepared at compounding \\ pharmacy before and after RDC 52/2011
}

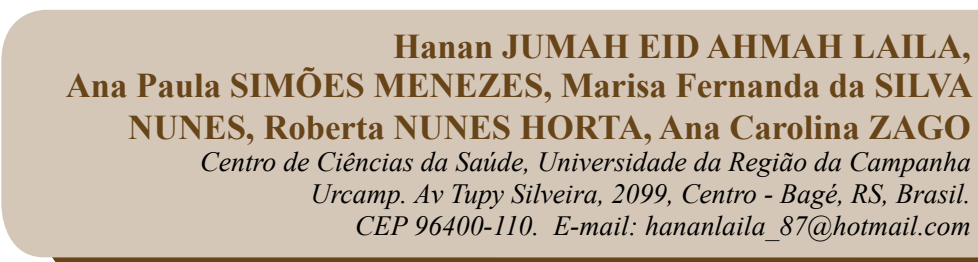

\section{ABSTRACT}

The aim of this study was to evaluate prescriptions of pharmaceuticals used to weight loss in a compounding pharmacy in the city of Pelotas - RS, before and after the term of the Anvisa RDC 52/2011. For this aim, prescriptions containing anorectic drugs were analyzed in the months from January to December in 2010 and 2012. The obtained results showed that women remain the gender that most consume these kind of drugs in both, 2010 and in 2012. In 2010 , the most prescribed anorectic were respectively amfepramone (68.7\%), femproporex $(26.2 \%)$, mazindol $(2.7 \%)$ and sibutramine $(2.4 \%)$, and the general practitioners were most prevalent physicians prescribing these drugs at both evaluated periods. In 2012, there was a considerable increase in the prescription of sibutramine. However, the validity of the RDC 52/2011 was effective to promote the reduction in prescriptions of anorectic drugs and it is noticeable the importance of regulatory acts on the part of health authorities, regulating the rational use of medicines.

Keywords: Obesity. Pharmacotherapy. Anorectics. Prescriptions.

\section{RESUMO}

O objetivo deste estudo foi avaliar o perfil de prescrições destinadas ao emagrecimento em uma farmácia de manipulação no município de Pelotas - RS, antes e após a vigência da RDC n $\mathrm{n}^{\mathrm{0}} 52 / 2011$. Para isso, foram analisados todos os receituários de controle especial contendo medicamentos anorexígenos entre os meses de janeiro a dezembro de 2010 e 2012. Os resultados obtidos demonstraram que o sexo feminino continua sendo o gênero que mais consome esses medicamentos tanto em 2010 quanto em 2012. No ano de 2010, os anorexígenos mais prescritos foram respectivamente anfepramona (68,7\%), femproporex $(26,2 \%)$, mazindol $(2,7 \%)$ e sibutramina $(2,4 \%)$, sendo os médicos da especialidade Clinica Geral aqueles que mais prescreveram em ambos os períodos avaliados.. Em 2012, foi observado um considerável aumento da prescrição de medicamento contendo o fármaco sibutramina. Entretanto, a vigência da $\mathrm{RDC} \mathrm{n}^{0}$ 52/2011 foi eficaz ao promover a redução da prescrição de anorexígenos e verifica-se a importância de Atos Regulatórios por parte das autoridades sanitárias, normatizando o uso racional de medicamentos.

Palavras Chave: Obesidade. Farmacoterapia. Anorexígenos. Prescrições 


\section{INTRODUÇÃO}

A obesidade é caracterizada pelo acúmulo excessivo de gordura pelo organismo, podendo causar desde o desenvolvimento de doenças graves não letais até a morte prematura, afetando diretamente a qualidade de vida dos indivíduos. É considerada um problema de saúde pública e sua incidência independe de fatores sócioeconomicos (1). A facilidade na obtenção de alimentos, a má-nutrição associada ao sedentarismo e os modos de vidas inadequados levam a um aumento excessivo da gordura corporal (2).

O tratamento da obesidade pode ser realizado por meio de medidas não medicamentosas, como dieta e exercícios físicos, uso de medicamentos ou ainda cirurgia (3). O tratamento farmacológico da obesidade é indicado para pacientes de ambos os sexos, com Índice de Massa Corporal (IMC) igual ou superior a $30 \mathrm{~kg} / \mathrm{m}^{2}$ que não tenham obtido resultados na perda de peso com mudanças no estilo de vida, e também para pacientes que tenham IMC entre 25 e $30 \mathrm{~kg} / \mathrm{m}^{2}$ que apresentem pelo menos uma comorbidade associada (4).

Os medicamentos destinados ao tratamento da obesidade devem possuir as seguintes características: reduzir o peso corporal e ter efeito benéfico sobre as doenças decorrentes do excesso de peso; ter efeitos colaterais toleráveis e/ou transitórios; apresentar eficácia e segurança mantidas a longo prazo; possuir mecanismo de ação conhecido; idealmente ter um custo razoável (5). Entretanto, nenhum dos fármacos atualmente apresenta todas essas características.

Os medicamentos que podem ser utilizados no processo de perda de peso são classificados em três grupos: fármacos que reduzem a absorção e digestão de nutrientes, representados particularmente pelo orlistat; fármacos que aumentam o gasto energético, como efedrina e cafeína; e fármacos que agem no sistema nervoso central, através dos neurotransmissores noradrenalina, serotonina e dopamina (6). Estes últimos são conhecidos como anorexígenos, sendo particularmente representados pelo femproporex, anfepramona, mazindol e sibutramina, sendo semelhantes quanto ao mecanismo de ação, efeitos clínicos, adversos e contra-indicações. Esses medicamentos atuam promovendo a diminuição do apetite e exercem ação estimulante central e no sistema cardiovascular (7).

Os anorexígenos não devem ser usados em associação com outros fármacos antidepressivos e induzem a tolerância, podendo levar à dependência física e psicológica, característica que impede o seu uso prolongado, cujo tratamento pode durar no máximo quatro meses. Seu uso é contraindicado durante a gestação, no período de lactação, a crianças com idade inferior a doze anos e também a pacientes com doenças cardiovasculares, uma vez que esses fármacos promovem a elevação da pressão arterial, taquicardia e palpitações (7).

Conforme a Comissão Internacional de Controle de Narcóticos (CICN), o Brasil foi o país que apresentou o maior consumo de medicamentos para emagrecer, havendo um aumento de $500 \%$ no consumo de anorexígenos no Brasil desde 1998 (8). Acredita-se que boa parte dos medicamentos usados para o tratamento da obesidade seja dispensada por farmácias de manipulação e se dá por via lícita através de prescrição médica (9).

O consumo elevado e abusivo de medicamentos para emagrecer levou à necessidade de efetivação de medidas reguladoras que possibilitam um maior controle sobre a venda e proporcionam uso racional de tais substâncias, de modo a evitar o uso danoso que os anorexígenos podem causar (4).

Por essa razão, a Anvisa (Agencia Nacional de Vigilância Sanitária), entre outras medidas implantadas com o propósito de manter um controle mais rígido na venda desses medicamentos, publicou no dia seis de outubro de 2011, a RDC $n^{\circ} 52$, dispondo sobre o cancelamento na venda de três anorexígenos - anfepramona, femproporex e mazindol - e impondo restrições na venda da sibutramina. De acordo com essa Resolução, a prescrição do medicamento sibutramina deverá vir acompanhada de um Termo de Responsabilidade do Prescritor preenchido, devidamente, em três vias (10).

Desse modo, o objetivo do estudo foi avaliar o perfil de prescrições de medicamentos anorexígenos aviadas por uma farmácia de manipulação no município de Pelotas - RS - antes e após a vigência da RDC $\mathrm{n}^{\circ} 52$ de 6 de outubro de 2011.

\section{MATERIAL E MÉTODOS}

Esta pesquisa foi realizada em uma farmácia de manipulação na região central do município de Pelotas, RS. A escolha desse estabelecimento comercial foi por conveniência, pela facilidade de acesso à mesma e pela disposição no fornecimento dos dados coletados.

Este estudo foi observacional, descritivo e retrospectivo realizado por meio do levantamento das prescrições de anorexígenos nos receituários de controle especial. Essas substâncias pertencem à classe de medicamentos controlados pela Portaria 344/98, fazendo parte dos receituários da lista $\mathrm{B} 2$, de cor azul, sendo referidos como psicotrópicos anorexígenos, sendo eles: anfepramona, femproporex, mazindol e sibutramina.

Tratou-se de um estudo quantitativo e qualitativo de utilização de medicamentos, conduzido entre os me- 
ses de janeiro a dezembro dos anos de 2010 e 2012. A escolha desses períodos justifica-se por ser anterior e posterior, respectivamente, à vigência da $\mathrm{RDC} \mathrm{n}^{\circ}$ 52/2011, podendo, dessa forma, avaliar os possíveis impactos dessa Resolução sobre a prescrição de drogas anorexígenas. $\mathrm{O}$ instrumento de coleta de dados continha questões fechadas caracterizado por uma planilha de transcrição de dados retirados a partir das vias de receituários médicos destinados ao emagrecimento (femproporex, anfepramona, mazindol e sibutramina) que ficaram retidas na farmácia de manipulação. As variáveis coletadas foram: especialidade do profissional prescritor, sexo do paciente e tipo de medicamento.

A pesquisa foi aprovada pelo Comitê de Ética em Pesquisa em humanos da Universidade da Região da Campanha, sob número de ata 0132012.

\section{RESULTADOS E DISCUSSÃO}

A partir da análise dos receituários de controle especial constatou-se que entre os meses de janeiro a dezembro de 2010 foram aviadas 4.396 prescrições de controle especial, 989 (22,49\%) contendo fármacos anorexígenos, sendo os mais prescritos representados na Tabela 1. Analisando-se os dados coletados referentes ao período compreendido entre os meses de janeiro a dezembro de 2012, ou seja, após a vigência da RDC $n^{\circ}$ 52/2011, observaram-se 2.666 prescrições contendo medicamentos de controle especial e apenas 169 (6,33\%) destes receituários continham substâncias com ação emagrecedora, sendo ela em sua totalidade o medicamento sibutramina (Tabela 1), e, quando comparados os dados referentes apenas ao total de prescrições de fármacos anorexígenos nos anos de 2010 e 2012, constatou-se que ocorreu uma redução de praticamente $83 \%$ no uso de medicamentos controlados destinados ao emagrecimento após a vigência da RDC n52/2011.

Tabela 1. Frequência prescrição de medicamentos controlados para o emagrecimento. Farmácia magistral.

Pelotas, RS. $2010(n=989)$ e $2012(n=169)$.

\begin{tabular}{|l|l|l|}
\multicolumn{1}{|c|}{ Fármaco } & \multicolumn{1}{c|}{2010} & \multicolumn{1}{c|}{$2012 *$} \\
\hline Anfepramona & $68,7 \%(679 / 989)$ & $* *$ \\
\hline Femproporex & $26,2 \%(259 / 989)$ & $* *$ \\
\hline Mazindol & $2,7 \%(27 / 989)$ & $* *$ \\
\hline Sibutramina & $2,4 \%(24 / 989)$ & $100 \%(169 / 169)$ \\
\hline
\end{tabular}

* Após a vigência da RDC n52/2011.

** Não foi observado consumo deste medicamento no período observado.

Contudo, pode ser observado que o consumo de sibutramina obteve um considerável aumento nos me- ses analisados do ano de 2012 (169/2.666, 6,3\%), quando comparado ao período anterior à RDC $\mathrm{n}^{\circ} 52 / 2011$ (24/4.396, 0,5\%) em 2010, constatando-se que a venda deste fármaco no ano de 2012 foi cerca de onze vezes superior ao ano de 2010.

A maior frequência de consumo de fármacos destinados ao emagrecimento foi entre o sexo feminino, sendo $81,9 \%(\mathrm{n}=810 / 989)$ no ano de 2010 e $86,9 \%$ ( $\mathrm{n}=147 / 169)$ no ano de 2012.

Ao analisar a especialidade médica que mais prescreveu fármacos anorexígenos, a Tabela 3 mostra uma ampla variedade de especialidades no ano de 2010. Após a vigência da $R D C n^{\circ} 52 / 2011$, pode ser observado que apenas clínicos gerais prescreveram o fármaco sibutramina (Tabela 2).

TABELA 2. Frequência da prescrição de anorexígenos de acordo com a especialidade médica. Farmácia magistral. Pelotas, RS.2010 (n=989) e 2012 ( $n=169)$

\begin{tabular}{|l|l|l|}
\multicolumn{1}{c|}{ Prescritor } & \multicolumn{1}{|c|}{2010} & \multicolumn{1}{c|}{$2012 *$} \\
\hline Clinico Geral & $69,0 \%(682 / 989)$ & $100 \%(169 / 169)$ \\
\hline Endocrinologista & $25,0 \%(247 / 989)$ & $* *$ \\
\hline Internista & $3,0 \%(30 / 989)$ & $* *$ \\
\hline Ortopedista & $0,9 \%(9 / 989)$ & $* *$ \\
\hline Psiquiatra & $0,7 \%(7 / 989)$ & $* *$ \\
\hline Outras & $1,4 \%(14 / 989)$ & $* *$ \\
\hline
\end{tabular}

* Após a vigência da RDC n52/2011.

** Não foi observada a especialidade médica prescrevendo anorexgenos no período observado

Confirmando a tendência de utilização de anorexígenos anterior ao período de proibição das mesmas, um estudo realizado por Noto et al. (2002) ressaltou que a maior parte dos medicamentos destinados à supressão do apetite eram dispensados por farmácias magistrais (11), sendo respectivamente maior a prevalência de anfepramona e femproporex, indo ao encontro do estudo realizado por Guimarães et al. (2004) no qual o anorexígeno mais consumido foi a anfepramona, corroborando com os dados obtidos no presente estudo (12).

Contudo, Silva \& Campesatto-Mella (2008), ao avaliarem o consumo de anorexígeno por acadêmicas de uma Instituição de Ensino Superior, verificaram que a sibutramina e o femproporex foram os fármacos de maior prevalência (13), sendo que esse fato estava relacionado à condição social das entrevistadas, visto que o maior consumo de sibutramina foi observado pelas acadêmicas com renda familiar acima de 7 salários mínimos, enquanto o uso desse medicamento entre as acadêmicas com renda familiar entre 2 e 3 salários mínimos foi nulo. 
Uma das principais causas para o consumo irracional de anorexígenos estava relacionada às distorções da imagem corporal devidas à busca pelo corpo perfeito e magro, difundido particularmente pela mídia o que provoca grande impacto no público feminino, o qual busca um padrão de beleza exigido pela sociedade e acaba adotando medidas drásticas para se adequar à esse padrão, recorrendo aos anorexígenos, inúmeras vezes sem acompanhamento médico adequado (13). No estudo realizado por Massuia et al. (2008), 29 usuários de fármacos anorexígenos pertenciam ao sexo feminino e apenas um era do sexo masculino (14). O mesmo foi relatado no estudo de Martins et al. (2012), onde 62,2\% consumidores de anorexígenos eram mulheres (16). Os resultados do presente estudo concordam com os autores acima indicados. Também se observou que, mesmo com a vigência da $\mathrm{RDC} \mathrm{n}^{\circ} 52 / 2011$, o padrão de consumo relacionado ao sexo do paciente não sofreu alterações; o gênero feminino continuou sendo o maior consumidor de medicamentos destinados à perda de peso. Uma das possíveis razões para a diferença de consumo desses medicamentos entre os diferentes gêneros provavelmente esteja relacionada ao grau de satisfação corporal entre os sexos, sendo que o público feminino apresenta maior índice de insatisfação.

Vários estudos com substâncias anorexígenas são realizados todos os anos e muitas dessas substâncias estão sendo retiradas do mercado por razões de segurança (15). Essa iniciativa visa garantir o acesso seguro desses medicamentos pela população (16).

Desse modo, o cancelamento do registro dos anorexígenos e, assim, a consequente proibição da comercialização dos mesmos já era uma realidade em outros países, como nos da Europa, onde a anfepramona, o femproporex e o mazindol foram retirados do mercado no ano de 2000 (16), e, no Brasil, isso só foi acontecer com a vigência da RDC nº52/2011 (10). Nos Estados Unidos, o femproporex nunca chegou a ser comercializado, pois não recebeu autorização do FDA (Food and Drug Admnistration) para ser vendido (16).

Os motivos para o elevado consumo de anorexígenos não eram apenas resultado do aumento da prevalência de obesidade e do sobrepeso, mas também estavam diretamente relacionados ao uso largamente disseminado dos mesmos no Brasil, causados pela multiplicidade de produtos farmacêuticos no mercado e sua maciça publicidade e ausência de conscientização a respeito dos danos desses medicamentos (9). De acordo com o relatório da CICN, o consumo per capita de anorexígenos vinha aumentado no Brasil, Austrália, República Checa e uma intervenção reguladora poderia reduzir o uso indiscri- minado de anorexígenos no Brasil, observando que Chile e Argentina reduziram a dispensação de anorexígenos em $90 \%$ por meio de medidas de controle (8), apoiando os resultados deste trabalho, visto que se constatou uma redução em $83 \%$ no consumo de anorexígenos quando comparado ao período anterior à sua proibição, assim, fica claro o impacto da RDC $\mathrm{n}^{0} 52 / 2011$ na realidade de uso de anorexígenos.

Entretanto, antes mesmo da RDC n⿳052/2011, a Anvisa já vinha adotando medidas a fim de garantir maior controle e fiscalização na venda de anorexígenos no Brasil, como a RDC no 27/2007 (17), RDC n n $^{\circ}$ 58/2007 (18) e posteriormente a RDC no 25/2010 (19) sendo que as mesmas já mostravam resultados positivos no controle da dispensação de anorexígenos, porém, ainda insuficientes.

Com base no acima exposto, Castro et al. (2012) avaliaram as consequências da RDC 58/2007 sobre a prescrição e dispensação de anorexígenos em drogarias e farmácias magistrais em Belém e perceberam uma redução significativa na distribuição dos anorexígenos em ambos os tipos de estabelecimentos comerciais (20). Assim como Lungwtz et al (2012), que observaram uma redução estatisticamente significativa no aviamento dos medicamentos anfepramona, femproporex e mazindol após a RDC n ${ }^{\circ} 58 / 2007$ no município de Sorocaba, SP, e uma queda, porém não significativa, no consumo de sibutramina, a qual estava mais relacionada à vigência da $\mathrm{RDC} \mathrm{n}^{\circ} 27 / 2007$, que garantiu maior controle de medicamentos controlados, inclusive os psicotrópicos anorexígenos, através do Sistema Nacional de Gerenciamento de Produtos Controlados (SNGPC) (21). Frente a isso, o presente estudo corrobora com os estudos acima citados e reafirma a importância de Atos Regulatórios por parte das autoridades sanitárias, ao promoverem a normatização do uso racional de medicamentos.

Contudo, contatou-se o aumento da prescrição do medicamento sibutramina após a vigência da RDC $n^{\circ}$ 52/2011, mesmo com a adoção de medidas restritivas para sua comercialização. Esse aumento é justificado justamente pela proibição da venda dos demais anorexígenos, anfepramona, femproporex e mazindol. Assim, a sibutramina tornou-se o único fármaco dessa classe que ainda pode ser utilizado para fins de emagrecimento.

Entretanto, contrariando os dados acima obtidos Vieiro \& Locateli (2012), ao fazerem um comparativo nas vendas da sibutramina após a RDC n ${ }^{\circ} 25 / 2010$ e 52/2011 em um município de Santa Catarina, observaram a redução da comercialização desse fármaco, onde os menores índices registrados na venda da sibutramina corresponderam ao período posterior à $\mathrm{RDC} \mathrm{n}^{\mathrm{o}} 52 / 2011$ (22). Os autores justificaram essa redução tanto pela vi- 
gência das resoluções de 2010 e 2011, quanto pelos estudos realizados que comprovaram os malefícios que esse fármaco pode provocar a longo prazo.

Em relação à sibutramina, as discussões atuais a respeito dos seus malefícios estão fundamentadas nos resultados do estudo clínico SCOUT (Sibutramine Cardiovascular Outcome Trial), que envolveu 10.744 pacientes obesos com doença cardiovascular preexistente, diabetes tipo 2, ou ambas as doenças e que faziam uso da sibutramina (15). O SCOUT demonstrou que, em relação ao placebo, a sibutramina promoveu controle da obesidade em apenas $2,5 \%$ dos pacientes que participaram do estudo e aumentou significativamente os efeitos colaterais graves em $16 \%$ dos casos, estes últimos ocasionados particularmente devido à ação simpaticomimética da sibutramina à nível cardiovascular, podendo ocasionar hipertensão arterial, taquicardia, infarto agudo do miocárdio e acidente vascular cerebral (23), assim os resultados desse estudo, demonstraram que o tratamento da obesidade com a sibutramina foi ineficaz em relação aos eventos cardiovasculares (15).

Com base nisso, inúmeros países como Estados Unidos, Canadá e os da comunidade Europeia proibiram o consumo do medicamento sibutramina (23). Atualmente, no Brasil a sibutramina continua a ser comercializada, entretanto os resultados do estudo SCOUT levaram a Anvisa a remanejar a sibutramina da lista de medicamentos $\mathrm{C} 1$ para a B2 através da $\mathrm{RDC} \mathrm{n}^{\circ}$ 13/2010 (24), estipular a Dose Diária Recomendada máxima da sibutramina e outros anorexígenos com a RDC $n^{\circ}$ 25/2010 (19) e posteriormente promoveu a restrição para a venda da sibutramina, entre outras medidas, por meio da RDC n52/2011 (10).

Partindo dessas informações, fica evidente que a atitude da Anvisa, particularmente através do advento da RDC $n^{\circ} 52 / 2011$, frente ao consumo irracional e desordenado dos anorexígenos foi fundamental para garantir uma redução do aviamento dos medicamentos psicotrópicos anorexígenos, entretanto, ainda não foi eficaz para promover a redução do consumo de sibutramina, muito pelo contrário, visto que este trabalho demonstrou que o consumo de sibutramina aumentou após a vigência da RDC $n^{\circ}$ 52/2011, mesmo havendo estudos científicos que relatam os malefícios que ela pode provocar particularmente a nível cardiovascular, podendo ocasionar sérios prejuízos à saúde dos pacientes.

Outra possível consequência da vigência da Resolução acima citada é a alteração em relação ao padrão de especialidade médica que mais emitem anorexígenos, observando que essa Resolução provocou modificações quanto à diminuição da variabilidade de especialidades médicas, o que pode induzir a uma prescrição mais racional desses medicamentos. Entretanto, os clínicos gerais continuam sendo os maiores prescritores de sibutramina, demonstrando que a utilização dos mesmos pode estar sendo realizada de maneira inadequada, visto que o tratamento para a redução do peso deveria ser conduzido por um endocrinologista, o que não foi observado no presente estudo.

Em relação ao período anterior à $\mathrm{RDC} \mathrm{n}^{0} 52 / 2011$, as especialidades médicas que mais prescreveram foram semelhantes a inúmeros outros relatos disponíveis na literatura, como o de Monteiro (2008) (25) e o de Feltrin et al. (2009) (26), nos quais os clínicos gerais e endocrinologistas foram as especialidades médicas que mais prescreveram anorexígenos.

\section{CONCLUSÃO}

A proporção do uso de fármacos anorexígenos antes da intervenção da Anvisa, era preocupante, visto que, na maioria dos casos, medicamentos contendo esses ativos eram utilizados de forma indiscriminada e muitos dos indivíduos não estavam dentro dos padrões de IMC necessários para o uso dos mesmos, uma vez que esses fármacos apresentam inúmeras contraindicações e efeitos colaterais, e inclusive causam dependência física e psíquica. É fundamental, primeiramente, o paciente buscar a redução de peso pelos métodos convencionais, como prática de exercícios físicos e restrição alimentar, visto que esses não possuem contraindicações.

Este estudo foi um dos pioneiros no Brasil ao relatar o impacto da RDC n ${ }^{\circ} 52 / 2011$ sobre a prescrição de anorexígenos, demonstrando que a mesma promoveu a redução da prescrição de medicamentos anorexígenos e também demonstrou certo receio pelos médicos especialistas na prescrição da sibutramina, percebendo-se um avanço a fim de evitar o uso desnecessário desses medicamentos pelos pacientes não obesos.

Em contrapartida, o consumo de sibutramina obteve um considerável aumento, quando comparado ao período anterior à proibição do consumo dos anorexígenos, mesmo com a adoção de medidas restritivas para sua venda. Entretanto, há necessidade da realização de mais estudos referentes às consequências da RDC n ${ }^{\circ} 52 / 2011$ sobre a prescrição de sibutramina, a fim de promover comparações com outras regiões do Brasil.

Vale ressaltar que a vigência desta Resolução restringiu muito as opções de tratamento farmacológico aos pacientes com obesidade mórbida ou àqueles que apresentam patologias associadas ao excesso de peso, cuja adoção de hábitos de vida mais saudáveis se mostrou 
insuficiente para garantir a redução do peso e melhorar a qualidade de vida.

Desse modo, acima de qualquer medida de restrição ou de proibição no uso de medicamentos, há necessidade da adoção de medidas educativas e conscientizadoras a respeito dos medicamentos usados para o tratamento da obesidade, a fim de evitar o uso indiscriminado e irracional pela população não obesa. É neste contexto que tanto o farmacêutico quanto o médico podem realizar um trabalho interdisciplinar a fim de atingir esse objetivo.

\section{REFERÊNCIAS}

1. Yano HM, Santos AP, Bugno A, Auricchio MT. Pesquisa de anorexígenos e benzodiazepínicos em formulações emagrecedoras e avaliação de rotulagem, em análises da Seção de Farmacognosia do Instituto Adolfo Lutz no período de junho de 2004 a março de 2007. Rev Inst Adolfo Lutz. 2008; 67(1):78-82.

2. Repetto G, Rizzolli J, Bonatto C. Prevalência, Riscos e Soluções na Obesidade e Sobrepeso: Here, There, and Everywhere. Arq Bras Endocrinol Metabol. 2003;47(6):633-35.

3. Wannmacher L. Ministério da Saúde. Obesidade Evidências e Fantasias. Revista Uso racional de medicamentos: temas selecionados. 2004; 1(3):01-06.

4. Carneiro MFG, Júnior AAG, Acurcio FA. Prescrição, dispensação e regulação do consumo de psicotrópicos anorexígenos em Belo Horizonte, Minas Gerais, Brasil. Cad Saúde Pública. 2008; 24(8):1763-72.

5. Mancini Mc, Halpern A. Tratamento Farmacológico da Obesidade. Arq Bras Endocrinol Metabol. 2002; 46(5):497-513.

6. Negreiros IIF, Oliveira DC, Figueredo MRO, Ferraz DLM, Souza LS, Moreira J et al. Perfil dos efeitos adversos e contraindicações dos fármacos moduladores de apetite: uma revisão sistemática. $\mathrm{Nu}$ trire: Rev Soc Bras Alimentação e Nutrição. 2011; 36(2):137-60.

7. Martins MCC, Filho MDS, Moura FS, Carvalho JSR, Moller $\mathrm{MC}$, Neves RV et al. Uso de drogas antiobesidade entre estudantes universitários. Rev Assoc Médica Bras. 2011; 57(5):570-76.

8. Internacional Narcotics Control Board (INCB) 2005. Relatório da Junta Internacional de Controle de Narcóticos em 2004, 1-99. ( www.incb.org/pdf/e/ar/2004/incb_report_2004_full. pdf). Acessado em 20 de setembro de 2013.

9. Melo CM, Oliveira DR. O uso de inibidores de apetite por mulheres: um olhar a partir da perspectiva de gênero. Ciência e Saúde Coletiva. 2011; 6(5):2523-32.

10. BRASIL. Ministério da Saúde. Agencia Nacional de Vigilância Sanitária. Resolução ${ }^{\circ} 52$, de 6 de outubro de 2011. Determina a proibição do uso das substâncias anfepramona, femproporex e mazindol, seus sais e isômeros, bem como intermediários e medidas de controle da prescrição e dispensação de medicamentos que contenham a substância sibutramina, seus sais e isômeros, bem como intermediários e dá outras providências. 2011.

11. Noto AR, Carlini EA, Mastroianni PC, Alves VC, Galduróz JCF, Kuroiwa $\mathrm{W}$ et al. Análise da prescrição e dispensação de medicamentos psicotrópicos em dois municípios do Estado de São Paulo, Brasil. Rev Bras Psiquiatria. 2002; 24(2):68-73.

12. Guimarães JL, Godinho PHG, Cruz R, Kappann JI, Junior LAT. Consumo de drogas psicoativas por adolescentes escolares de Assis, SP. Rev Saúde Pública. 2004;38(1):130-32.

13. Silva M, Campesatto-Mella EA. Avaliação do uso de anorexígenos por acadêmicas de uma instituição de ensino superior em Maringá, PR. Arq Ciências da Saúde Unipar. 2008; 12(1):43-50.

14. Massuia GR, Bruno TIB, Silva VL. Regime de emagrecimento $x$ utilização de drogas. Rev Científica UNIFAE. 2008;2: 5-9.
15. Paumgartten FJR. Benefícios dos inibidores de apetite à saúde a longo prazo permanecem sem comprovação. Rev Saúde Pública. 2011:45(6):1192-1196.

16. Martins ELM, Amaral MPH, Ferreira MBC, Mendonça AE, Pereira MCS, Pereira DC, Pereira PM, Brumano LP. Dispensações de psicotrópicos anorexígenos no município de Juiz de Fora, Minas Gerais, Brasil. Rev Ciência \& Saúde Coletiva. 2012: 17(12):3331-3342.

17. BRASIL. Ministério da Saúde. Agencia Nacional de Vigilância Sanitária. Resolução no 27 , de 30 de março de 2007. Dispõe Sobre o Sistema Nacional de Gerenciamento de Produtos Controlados - SNGPC, estabelece a implantação do módulos em Drogarias e Farmácias. 2007.

18. BRASIL. Ministério da Saúde. Agencia Nacional de Vigilância Sanitária. Resolução $\mathrm{n}^{\circ} 58$, de 5 de setembro de 2007. Dispõe sobre o aperfeiçoamento do controle e fiscalização de substâncias psicotrópicas anorexígenas e dá outras providências. 2007.

19. BRASIL. Ministério da Saúde. Agencia Nacional de Vigilância Sanitária. Resolução ${ }^{\circ}$ 25, de 30 de junho de 2010. Altera a RDC $\mathrm{N}^{\circ} 58$, de 5 de setembro de 2007 , que dispõe sobre o aperfeiçoamento do controle e fiscalização de substâncias psicotrópicas anorexígenas e dá outras providências. 2010.

20. Castro LVS, Farias Junior G, Teixeira FM, Vieira JRS, Maia CSF. Impact of health legislation on the sale of anorectics in a city in the Amazon region. Acta Amazonica. 2012:42(2): 215 - 220.

21. Lungwtz P, Martins E, Gerenutti M. Influência de Atos Regulatórios sobre a manipulação de substâncias psicotrópicas anorexígenas. Rev Saúde \& Transformação Social. 2012: (3): 2: 52-58.

22. Vieiro TR, Locatelli C. Perfil de prescrição e comparativo de vendas de sibutramina antes e após RDC $\mathrm{n}^{\circ} 25$ de 30 de junho de 2010 e RDC n 52 de 06 de outubro de 2011 em uma farmácia da cidade de Videira - SC. Rev Interd Estudos em Saúde: 2012:2(1):8-17.

23. Casulari LA, Motta LDC. Reflexões sobre os conflitos de interesses entre médicos e a indústria farmacêutica: a propósito da sibutramina. Brasília Médica. 2011: 48(3):308-313.

24. BRASIL. Ministério da Saúde. Agencia Nacional de Vigilância Sanitária. Resolução nº 13, de 26 de março de 2010. Dispõe sobre a atualização do Anexo I, Listas de Substâncias Entorpecentes, Psicotrópicas, Precursoras e Outras sob Controle Especial, da Portaria SVS/MS n 344 , de 12 de maio de 1998 e dá outras providências. 2010.

25. Monteiro ARM. Produtos à base de plantas dispensados em ervanárias para o emagrecimento: efeitos terapêuticos, toxicologia $\mathrm{e}$ legislação. [Dissertação de Mestrado]. Porto, Portugal: Universidade do Porto; 2008.

26. Feltrin AC, Zordan G, Wagner F, Schmitt GC, Boligon AA, Delamolle $\mathrm{N}$ et al. Medicamentos anorexígenos - panorama da dispensação em Farmácias comerciais de Santa Maria (RS). Saúde. $2009 ; 35(1): 46-51$. 